\title{
A stochastic model for operating room planning under uncertainty and equipment capacity constraints
}

\author{
J. Razmi • M. Barati • M. S. Yousefi • \\ J. Heydari
}

Received: 3 September 2014/ Accepted: 8 January 2015/Published online: 5 February 2015

(C) The Author(s) 2015. This article is published with open access at Springerlink.com

\begin{abstract}
In the present economic context, the operating theater is considered as a critical activity in health care management. This paper describes a model for operating room (OR) planning under constraint of a unique equipment. At first level we schedule elective surgeries under the uncertainty of using a unique equipment. At the second level we consider emergency surgeries, and at the third level a coefficient factor for surgeons is introduced in using this unique equipment. The planning problem consists in scheduling a unique equipment and assigning elective cases to different periods over a planning horizon to minimize the sum of elective patient related costs and overtime costs of ORs. The most important factor that we have focused on this paper is equipment resource constraint. A new mathematical programming model is first proposed and at the second and third level, a new stochastic mathematical programming model is proposed. Then sample average approximation is presented to approximate the problem with sample size $\mathrm{N}$ and then Lingo is used as an exact approach. Because of NP-hardness, exact method does not work for large size problems, so a Metaheuristic approach (differential evolution) is proposed for large size problems. Numerical results show that important gains (approximately $3.5 \%$ in overall cost) can be realized by this stochastic OR planning model.
\end{abstract}

School of Industrial Engineering, College of Engineering,

University of Tehran, Tehran, Iran

e-mail: jrazmi@ut.ac.ir

M. S. Yousefi

Department of Mechanical Engineering, Malek e Ashtar

University of Technology, Shahin Shahr, Isfahan, Iran
Keywords Mathematical programming model - Unique Equipment · Operating rooms · Surgery planning · Differential evolution (DE) - Sample average approximation (SAA)

\section{List of symbols}

$H$

$t=1,2, \ldots, H$

K

$T_{t}$

$H_{t}^{\prime}=1,2, \ldots,\left(\frac{60 * T_{t}}{K}\right)$

$j=1,2, \ldots, H_{t}^{\prime}$

$N$

$i=1, \ldots, N$

$P_{i}$

$B_{i}$

$\mathrm{CE}_{i t}$

$\mathrm{CUE}_{i t}$

$\mathrm{CO}_{t}$

$m_{i}$

$q_{i}$

$w_{t}$
Planning horizon

Time period index

C-ARM performance duration in each use

Total available regular capacity of all ORs in period $t$

Number of C-ARM performance opportunities in each period Frequency of using the equipment in each period

Number of elective cases

Elective case index

Time needed for performing elective case $i$ which is assumed to be a given constant

Earliest period for performing case $i$ Cost of performing elective case $i$ in period $t$ for $t=B_{i}, B_{i+1}, \ldots, B_{H+1}$ Cost of using C-ARM for patient $i$ in period $t$ for $t=1, \ldots, H$ Cost per unit of overtime in period $t$ Number of available $J$ in each interval of performing C-ARM (i.e., C-ARM free time, between two performance for one patient) Frequency of using C-ARM for elective case $i$

Capacity needed for emergency cases of period $t$ 
$d_{i}$

C-ARM performance duration, it is assumed to be factor of $K$ (surgeon's coefficient factor)

\section{Introduction}

Surgery is one of the most important functions in hospitals and it generates revenue and admissions to hospitals. The operating cost of a surgery department is one of the largest hospital cost category, approximately one-third of the total cost (Macario et al. 1995). While surgery is the largest cost center, it also accounts for approximately two-third of hospital revenues (Jackson 2002). Therefore, small improvements in efficiency could translate into significant savings and benefits to the patient by early treatment and reducing the hospitalization cost as well as the hospital. For these reasons, effectively managing the surgical resources reduce the costs which this draws considerable attention from the healthcare community. Sometimes the budget of hospital is fixed and the goal is to maximize the number of cured cases under a fixed budget (Tao et al. 2012)

In this paper, we focus on planning a unique equipment (C-Arm imaging equipment) of operating rooms (ORs) for elective and Emergency surgeries. Equipment plays a vital role in hospitals and especially ORs, equipment resources are key sources in hospitals and their shortage or unavailability causes a lot of problems and many performances and treatments will stop. These problems may lead to death of the patient. Indeed it is very essential to plan and schedule hospital's equipment. Scheduling and planning equipment seems more important when the equipment is unique and also very useful. The main aspect of this study is to schedule this unique equipment for elective and emergency patients. Electives cases can be delayed and planned for future dates. The planning of surgical activities in ORs has been extensively addressed over the past three decades. Magerlein and Martin (1978) presented a review of surgical suite scheduling and discussed procedures for planning patients in advance of their surgical dates and techniques for the assignment of patients to ORs at specific times of day. Gerchak et al. (1996) propose the use of a stochastic dynamic program to determine the elective surgeries that may be performed each day according to the required procedures of that day. Marcon et al. (2003) model the problem of allocating operations to ORs as a multiple knapsack problem while minimizing the difference of workload between the rooms and minimizing the risk of no-shows. They assume that the date of the surgical procedures is known. Agnetis et al. (2012) investigated long-term policies for determining the Master Surgical

Schedule (MSS) throughout the year. Guinet and Chaabane (2003) heuristically solve the assignment problem of operations to ORs with resource capacity and time-window additive constraints by an extension of the Hungarian method. They minimize a fixed patient intervention cost based on the hours of activity of an OR and the patient waiting time. For scheduling surgical procedures, Kharraja et al. (2003) consider operating and recovery rooms each with $\mathrm{k}$ beds and model them as $\mathrm{k}$ flow shops. Chaabane (2004) treats the operating and recovery rooms as a hybrid flow shop with recirculation. Lakshimi and SivaKumarAppa (2013) provided sufficient information to analysts who are interested in using queuing theory to model a health care process and who want to locate the details of relevant models. Alkhabbaz and M'Hallah (2013) propose a mixed integer program to solve the mathematical model for the case of a specific health care unit using an off-theshelf optimizer to investigate the problem of designing timetables for nurses working in Kuwaiti health care units that operate around the clock. However, all these approaches do not account equipment shortage, which is a very prevalent problem in most of the hospitals (especially hospitals with financial difficulties). Dexter et al. (1999a, b) used online and off-line bin-packing techniques to plan elective cases and evaluated their performances using simulation. But none of the mentioned papers had noticed the important role of such unique equipment in hospitals.

The goal of this paper is to develop an optimization model and algorithms (exact and metaheuristic) for elective and emergency surgeries planning in ORs with uncertain demand for using a unique equipment. This device is portable and it can be used for all of the ORs in an operating theater.

C-Arm is one of the expensive devices with difficult maintenance, so it is most of the time unique in most of the hospitals, in this case study research, C-Arm is unique.

A mobile $\mathrm{C}$-arm is a medical imaging device that is based on X-ray technology and can be used flexibly in various ORs within a clinic. The name is derived from the C-shaped arm used to connect the X-ray source and X-ray detector to one another.

Mobile imaging systems are an essential part of everyday hospital life: Specialists in fields such as surgery, orthopedics, traumatology, vascular surgery and cardiology use $\mathrm{C}$-arms for intraoperative imaging.

The devices provide high-resolution $\mathrm{X}$-ray images in real time, thus allowing the physician to monitor progress at any point during the operation and immediately make any corrections that may be required. Consequently, the treatment results are better and patients recover more quickly. Hospitals benefit from cost savings through fewer follow-up operations and from minimized installation efforts. C-Arm is shown in Fig. 1. 
A C-arm comprises a generator (X-ray source) and an image intensifier or flat-panel detector. The C-shaped connecting element allows movement horizontally, vertically and around the swivel axes, so that X-ray images of the patient can be produced from almost any angle.

The generator emits X-rays that penetrate the patient's body. The image intensifier or detector converts the X-rays into a visible image that is displayed on the $\mathrm{C}$-arm monitor. The doctor can identify and check anatomical details on the image such as blood vessels, bones, kidney stones and the position of implants and instruments at any time.

The problem consists of determining a plan that specifies the set of elective cases that would be performed in each period over a planning horizon with considering the uncertain demand of emergency cases and also uncertain demand for $\mathrm{C}$-arm as a unique equipment. In this scheduling we should consider to have no overlap in using this unique equipment for different surgeries. Overlaps cause cancellation and also make surgeries longer than usual that increase patient related costs. When this unique equipment is being used for one patient, other patients should wait for it, this will increase the waiting time if not scheduled. This waiting time increases the costs of patient as well as hospital, so we should try to omit these waiting times. But financial aspects alone are not sufficient in establishing surgical schedules. We can no longer ignore the significant place of the people involved in the medical process execution. Thus, in addition to the usual economic objective presented in the literature we undertake human dimension. The surgery plan should minimize costs related to the overutilization of ORs and costs related to performing elective

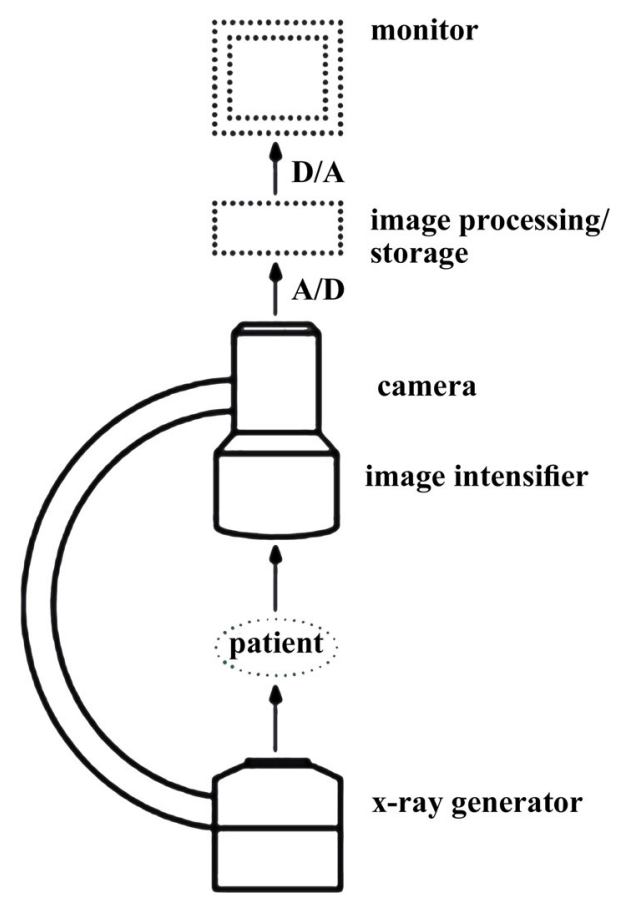

Fig. 1 C-Arm image 1 surgery. Although numerous studies show the extreme importance of accounting uncertainties such as emergency demand in OR planning and demand for this unique equipment, existing OR planning approaches all use deterministic optimization models and assume that the hospital uses dedicated ORs to serve emergency patients, or devotes a fixed portion of OR capacity to perform only the emergency surgeries. The main contributions of this paper include (1) a new OR planning model that explicitly takes into account elective and emergency patients under uncertainty of using a unique equipment (C-ARM), (2) model's Np-hardness causes using Metaheuristic method for solving the problem in large size problems. Our OR planning method shows a good reduction of overall cost. The remainder of this paper is organized as follows: "A programming model for scheduling OR elective operations" section presents the planning model for the problem. In section "Solution strategy: sample average approximation" sample average approximation is presented to approximate the problem with sample size $N$. Metaheuristic method is introduced in section "The proposed Metaheuristic method"; Numerical results of the optimization method are presented in section "Computational experiments". "Conclusions and future research" section concludes the paper and discusses possible extensions of this work.

\section{A programming model for scheduling OR elective operations}

This work concerns the planning of elective surgery at a hospital surgical suite over a planning horizon $\mathrm{H}$ with considering the constraint of having a unique equipment. The surgical suite capacity is dedicated to elective cases that are to be planned in advance; there are $N$ requests for elective surgery. Each elective case $i(i=1, \ldots, N)$ has the following characteristics:

- $p_{i}$, the time needed for performing elective case $i$, which we call operating time, and includes not only the surgery time but also setup time, cleaning, etc.;

- $B_{i}$, the release period

Accurate estimates of operating times are necessary to have efficient OR planning. Shukla et al. (1990) recommend using historical information to estimate the operating time of elective cases. Zhou and Dexter (1998) advocate the use of log-normal distributions to approximate surgery durations. Surgeons and OR managers can also provide good estimations of operating times. In this work, we assume that operating times of all elective cases are known and deterministic, because all of these data are collected from Isfahan Kashani Hospital. The release date $B_{i}(i=1, \ldots, N)$ is the earliest period at which elective case $i$ can be performed, it may represent hospitalization date, date of medical test delivery, etc. $H_{t}^{\prime}$ counts the opportunities of using C-ARM in each 
period, for example if the period is $8 \mathrm{~h} \times 60=480 \mathrm{~min}$, and each performance time of C-ARM in each use is about $20 \mathrm{~min}$ (according to collected data) so $\mathrm{H}_{t}^{\prime}$ is $480 / 20=24$ opportunity for C-ARM performance.

For each elective case $i$ we define a set of costs $\mathrm{CE}_{i t}$ $\left(t=B_{i}, \ldots, H, H+1\right)$ and $\mathrm{CUE}_{\mathrm{it}}(t=1, \ldots, H)$. The $\mathrm{CE}_{\mathrm{it}}$ represents the cost of performing elective case $i$ in period $t$ and $\mathrm{CUE}_{i t}$ represents the cost of using C-ARM for those patients that need C-ARM. The period $H+1$ is added to the planning horizon for cases that are rejected from the current planning horizon and that will be considered in the next horizon. The cost structure proposed in this paper is fairly general. It can represent hospitalization costs (Jebali et al. 2005; Guinet and Chaabane 2003), penalties for waiting to get on schedule (Gerchak et al. 1996), optimal surgery date, patients' or surgeons' preferences, and eventual deadlines. For example, if case $i$ must be performed before period $\mathrm{Li}$, this constraint can be taken in account by choosing large costs $\mathrm{CE}_{i t}$ for $t>\mathrm{Li}$.

At the planning level, we are interested in determining a plan that specifies the set of elective cases to be performed in each period over the planning horizon. The assignment to a specific OR and the starting time of each case can be made at a later stage on a period-to-period basis (Weiss 1990; Denton and Gupta 2003). We assumed that ORs are identically equipped, each surgical case can be assigned to any OR, and only the total available capacity of all ORs (Operating theater capacity) is accounted. Let $T_{t}$ be the total available regular OR capacity in period $t$ in operating theater. If planned elective cases exceed this regular capacity, overtime costs are incurred. Let $\mathrm{CO}_{t}$ be the cost per unit of overtime in period $t$.

\section{Decision variables}

$X_{i t}=\{0,1\}$ with $X_{i t}=1$ if elective case $i$ is performed in period $t$ and 0 otherwise with $X_{i, H+1}=1$ if elective case $i$ is rejected in the current planning horizon.

$y_{i j t}=\{0,1\}$ with $y_{i j t}=1$ if elective case $i$ is performed with C-ARM in $J$ and period $t$.

Mathematical model

$$
\begin{aligned}
\operatorname{Minimize} J(x)= & \sum_{i=1}^{N} \sum_{T=B_{i}}^{H+1} \mathrm{CE}_{\mathrm{it}} x_{\mathrm{it}}+\sum_{t=1}^{H} \mathrm{CO}_{t} \mathrm{O}_{t} \\
& +\sum_{i=1}^{N} \sum_{j=1}^{H^{\prime \prime} t} \sum_{t=1}^{H} \mathrm{CUE}_{\mathrm{ijt}} y_{\mathrm{ijt}} \\
\text { subject to } \quad O_{t}= & {\left[\left(\sum_{i=1}^{N} P_{i} X_{i t}-T_{t}\right)^{+}\right], \quad \forall t=1, \ldots, H }
\end{aligned}
$$

$$
\begin{aligned}
& \sum_{T=B_{i}}^{H+1} x_{i t}=1 \quad \forall i=1, \ldots, N \\
& X_{i t}=\{0,1\}, \quad \forall i=1, \ldots, N, \quad \forall 0=1, \ldots, H+1 \\
& y_{i j t} \leq x_{i t} \quad \forall i=1, \ldots, N, t=1, \ldots, H, j=1, \ldots, H_{t}^{\prime} \\
& \sum_{j=1}^{H_{t}^{\prime}} y_{i j t}=q_{i} x_{i t} \quad \forall i=1, \ldots, N, t=1, \ldots, H \\
& \left(m_{i}+1\right) y_{i j t} * y_{i l t} \leq(j-l) y_{i j t} * y_{i l t} \quad \forall i=1, \ldots, N, \\
& t=1, \ldots, H, l=1, \ldots, H_{t}^{\prime}-1, j=l+1, \ldots, H_{t}^{\prime} \\
& (j-l) y_{i j t} * y_{i l t} \leq\left(m_{i}+1\right) *\left(q_{i}-1\right) \quad \forall i=1, \ldots, N, \\
& t=1, \ldots, H, l=1, \ldots, H_{t}^{\prime}-1, j=l+1, \ldots, H_{t}^{\prime} \\
& y_{i j t}=\{0,1\}
\end{aligned}
$$

The objective function (1) minimizes the expected overtime costs as well as elective cases related costs (waiting time costs, hospitalization costs, C-ARM performance cost, etc.). Constraints (2) estimate the expected overtime $O_{t}$ in each period.

Constraints (3) ensure that each elective case is assigned once and only once. Constraints (4) are the integrity Constraints. Constraint (5) ensures that if patient $i$ is not assigned, C-ARM will not be assigned to him/her, also. Constraint (6) is number of C-ARM performance for patient $i$. Constraints $(7,8)$ ensure that the interval duration (when C-ARM has been free after first performance for one patient to the next performance for mentioned patient) has a certain limitation.

\section{Emergency cases stochastic model}

This model can be extended to consider emergency patients. Emergency cases arrive randomly and have to be performed on the day of arrival. With considering emergency cases, planning problem becomes a stochastic problem. Emergency cases arrive randomly and must be served immediately on the day of their arrival. Equivalently, emergency cases arriving in a given time period are performed in the same period whatever the available capacity. Let $W_{t}$ be the total OR time needed for emergency cases arriving in period $t$. It is a stochastic parameter.

$w_{t}$ : Capacity needed for emergency cases of period $t$

$w_{t}$ is capacity needed for emergency cases of period $t$. The average daily capacity $\mathrm{E}\left[W_{t}\right]$ needed for emergency cases depends on the number of available ORs, because $W_{t}$ is normally distributed, mean and deviation standard of normal distribution is used. 


$$
\begin{aligned}
\operatorname{Minimize} J(x)= & \sum_{i=1}^{N} \sum_{T=B_{i}}^{H+1} \mathrm{CE}_{i t} x_{i t}+\sum_{t=1}^{H} \mathrm{CO}_{t} \mathrm{O}_{t} \\
& +\sum_{i=1}^{N} \sum_{j=1}^{H^{\prime \prime} t} \sum_{t=1}^{H} \operatorname{CUE}_{i j t} y_{i j t} \\
\text { Subjected to } \quad O_{t} & =E_{w t}\left[\left(W_{t}+\sum_{i=1}^{N} P_{i} X_{i t}-T_{t}\right)^{+}\right], \forall t \\
& =1, \ldots, H
\end{aligned}
$$

$$
\begin{aligned}
& \sum_{T=B_{i}}^{H+1} x_{i t}=1 \quad \forall i=1, \ldots, N \\
& X_{i t}=\{0,1\}, \quad \forall i=1, \ldots, N, \forall 0=1, \ldots, H+1 \\
& y_{i j t} \leq x_{i t} \quad \forall i=1, \ldots, N, t=1, \ldots, H, j=1, \ldots, H_{t}^{\prime} \\
& \sum_{j=1}^{H_{t}^{\prime}} y_{i j t}=q_{i} x_{i t} \quad \forall i=1, \ldots, N, t=1, \ldots, H \\
& \left(m_{i}+1\right) y_{i j t} * y_{i l t} \leq(j-l) y_{i j t} * y_{i l t} \quad \forall i=1, \ldots, N, \\
& t=1, \ldots, H, l=1, \ldots, H_{t}^{\prime}-1, j=l+1, \ldots, H_{t}^{\prime} \\
& (j-l) y_{i j t} * y_{i l t} \leq\left(m_{i}+1\right) *\left(q_{i}-1\right) \quad \forall i=1, \ldots, N, \\
& t=1, \ldots, H, l=1, \ldots, H_{t}^{\prime}-1, j=l+1, \ldots, H_{t}^{\prime} \\
& y_{i j t}=\{0,1\}
\end{aligned}
$$

The objective function (10) minimizes the expected overtime costs as well as elective cases related costs (waiting time costs, hospitalization costs, C-ARM performance cost, etc.). Constraints (11) estimate the expected overtime $O_{t}$ in each period.

Constraints (12) ensure that each elective case is assigned once and only once. Constraints (13) are the integrity Constraints. Constraint (14) ensures that if patient $i$ is not assigned, C-ARM will not be assigned to him, also. Constraint (15) is number of C-ARM performance for patient $i$. Constraints $(16,17)$ ensure that the interval duration (when C-ARM has been free after one performance for one patient to the next performance for that patient) has a certain limitation.

The elective case planning model (10-18) is a stochastic combinatorial problem and its NP-hardness is proved by Lamiri et al. (2008) and the NP-hardness remains true even for the two-period problem.

Surgeon's coefficient factor stochastic model

In such kind of operations where C-ARM is needed, for having a better prediction of length of operations, we introduce a coefficient factor for surgeons in using this equipment. Most of the time it depends on surgeon's experience, i.e., talented and experienced surgeons are more talented in using this equipment and they find the target easier and sooner, in this case study this equipment was used for the same part of the same operation by three surgeons, the recorded durations were 10,17 and $23 \mathrm{~min}$. It is obvious that for having a more precise scheduling we should introduce a coefficient factor for surgeons in using this equipment.

$d_{i}$ : C-ARM performance duration, it is assumed to be factor of $K$ (surgeon's coefficient factor)

For $(J)$ constraints $(16,17)$ change to the following constraints;

$$
\begin{gathered}
\left(m_{i}+d_{i}\right) y_{i j t} * y_{i l t} \leq(j-l) y_{i j t} * y_{i l t} \quad \forall i=1, \ldots, N \\
t=1, \ldots, H, l=1, \ldots, H_{t}^{\prime}-1, j=l+1, \ldots, H_{t}^{\prime} \\
(j-l) y_{i j t} * y_{i l t} \leq\left(m_{i}+d_{i}\right) *\left(q_{i}-1\right) \quad \forall i=1, \ldots, N \\
t=1, \ldots, H, l=1, \ldots, H_{t}^{\prime}-1, j=l+1, \ldots, H_{t}^{\prime}
\end{gathered}
$$

\section{Solution strategy: sample average approximation}

The models presented in (2.4 and 2.5) are stochastic and strongly Np-hard (Lamiri et al. 2008). We propose a solution strategy for these stochastic models. By Shapiro et al. (2002), the optimal solution of the SAA problem provides an exact optimal solution of the true $J$ (i.e., model 1-9) with probability one (w.p 1) for a sample size $N$ that is large enough. Moreover, Shapiro and Homem-de-Mello (2001) show that the probability of providing an exact optimal solution of the true problem approaches one exponentially fast as $N$ tends to infinity. These results imply that a good approximate solution can be obtained with a relatively small sample size. The following mathematical model describes the SAA problem of the $J$ with sample size $N$. $N$ independent random samples $W_{1 t}, \ldots, W_{n t}$ are generated for each random variable $W_{t}, t \in(1, \ldots, H)$, and the mathematical expectations in the objective function (1) are approximated by their sample averages (empirical means). Consequently, the true problem $(J)$ can be approximated by a sample average approximation (SAA):

$$
\begin{aligned}
J * & =\operatorname{Minimize} J(x) \\
& =\sum_{i=1}^{N} \sum_{T=B_{i}}^{H+1} \mathrm{CE}_{i t} x_{i t}+\sum_{t=1}^{H} \mathrm{CO}_{t} \mathrm{O}_{t}+\sum_{i=1}^{N} \sum_{j=1}^{H^{\prime \prime} t} \sum_{t=1}^{H} \mathrm{CUE}_{i j t} y_{i j t}
\end{aligned}
$$

Subject to $O_{t k}>W_{t k}+\sum_{i=1}^{N} p_{i} X_{i t}-t_{t}, \quad \forall t=1, \ldots, H$,

$$
\begin{aligned}
& \forall k=1, \ldots ., K, \\
& O_{t}=\frac{\sum_{k=1}^{k} O_{t k}}{K}, \quad \forall t=1, \ldots, H,
\end{aligned}
$$


$\sum_{t=b_{i}}^{H+1} X_{i t}=1, \quad \forall i=1, \ldots, N$,

(Binary variables) $X_{i t}=\{0,1\} \forall i=1, \ldots, N$,

$$
\forall t=1, \ldots, H+1
$$

(Real variables) $O_{t k} \geq 0, \forall t=1 \ldots, H \forall k=1, \ldots, K$,

$y_{i j t} \leq x_{i t} \quad \forall i=1, \ldots, N, t=1, \ldots, H, j=1, \ldots, H_{t}^{\prime}$

$\sum_{j=1}^{H_{t}^{\prime}} y_{i j t}=q_{i} x_{i t} \quad \forall i=1, \ldots, N, t=1, \ldots, H$

$\left(m_{i}+d_{i}\right) y_{i j t} * y_{i l t} \leq(j-l) y_{i j t} * y_{i l t} \quad \forall i=1, \ldots, N$,

$t=1, \ldots, H, l=1, \ldots, H_{t}^{\prime}-1, j=l+1, \ldots, H_{t}^{\prime}$

$(j-l) y_{i j t} * y_{i l t} \leq\left(m_{i}+d_{i}\right) *\left(q_{i}-1\right) \quad \forall i=1, \ldots, N$,

$=1, \ldots, H, l=1, \ldots, H_{t}^{\prime}-1, j=l+1, \ldots, H_{t}^{\prime}$

\section{The proposed Metaheuristic method}

The planning problems can be solved by lingo optimization method (exact solution), but cannot be found in a reasonable amount of time for problems of large size. Therefore, Metaheuristic methods seem to be an interesting choice to be investigated. In this paper, we propose DE [differential evolution (DE)] algorithm. DE is used for multidimensional real-valued functions but does not use the gradient of the problem being optimized, which means DE does not require for the optimization problem to be differentiable as is required by classic optimization methods such as gradient descent and quasi-Newton methods. DE can therefore also be used on optimization problems that are not even continuous, are noisy, change over time, etc. Rocca et al. (2011). DE optimizes a problem by maintaining a population of candidate solutions and creating new candidate solutions by combining existing ones according to its simple formulae, and then keeping whichever candidate solution has the best score or fitness on the optimization problem at hand. In this way the optimization problem is treated as a black box that merely provides a measure of quality given a candidate solution and the gradient is therefore not needed. Variants of the DE algorithm are continually being developed in an effort to improve optimization performance. Many different schemes for performing crossover and mutation of agents are possible in the basic algorithm given above; see, e.g., (Storn 1996). More advanced DE variants are also being developed with a popular research trend being to perturb or adapt the DE parameters during optimization, see, e.g., Price et al.
(2005), Liu and Lampinen (2005), Qin and Suganthan (2005), Civicioglu (2011) and Brest et al. (2006).

A basic variant of the DE algorithm works by having a population of candidate solutions (called agents). These agents are moved around in the search space by simple mathematical formulae to combine the positions of existing agents from the population. If the new position of an agent is an improvement it is accepted and forms part of the population, otherwise the new position is simply discarded. The process is repeated and by doing so it is hoped, but not guaranteed, that a satisfactory solution will eventually be discovered.

Formally, let $f: \mathbb{R}^{n} \rightarrow \mathbb{R}$ be the cost function which must be minimized or fitness function which must be maximized. The function takes a candidate solution as argument in the form of a vector of real numbers and produces a real number as output which indicates the fitness of the given candidate solution. The gradient of $f$ is not known. The goal is to find a solution $m$ for which $f(m) \leq f(p)$ for all $p$ in the search space, which would mean $m$ is the global minimum. Maximization can be performed by considering the function $h:=-f$ instead.

Let $\mathbf{x} \in \mathbb{R}^{n}$ designate a candidate solution (agent) in the population. The basic DE algorithm can then be described as follows:

- Initialize all agents $\mathbf{x}$ with random positions in the search space.

- Until a termination criterion is met (e.g., number of iterations performed, or adequate fitness reached), repeat the following:

For each agent $\mathbf{x}$ in the population do:

- Pick three agents a, b, and $\mathbf{c}$ from the population at random, they must be distinct from each other as well as from agent $\mathbf{x}$

- Pick a random index $R \in\{1, \ldots, n\}$ ( $n$ being the dimensionality of the problem to be optimized).

- Compute the agent's potentially new position $\mathbf{y}=\left[y_{1}\right.$, $\ldots, y_{n}$ ] as follows:

- For each $i$, pick a uniformly distributed number $r_{i} \equiv U(0,1)$

- If $r_{i}<\mathrm{CR}$ or $i=R$ then set $y_{i}=a_{i}+F \times\left(b_{i}-c_{i}\right)$ otherwise set $y_{i}=x_{i}$

- In essence, the new position is outcome of binary crossover of agent $\mathrm{x}$ with intermediate agent $\mathbf{z}=\mathbf{a}+F \times(\mathbf{b}-\mathbf{c})$.

- If $f(\mathbf{y})<f(\mathbf{x})$ then replace the agent in the population with the improved candidate solution, that is, replace $\mathbf{x}$ with $\mathbf{y}$ in the population. 
- Pick the agent from the population that has the highest fitness or lowest cost and return it as the best found candidate solution.

\section{Computational experiments}

This section presents numerical results of the "exact" (lingo) and Metaheuristic (DE) optimization method. All solution methods have been implemented in MATLAB2009. The numerical experiments are carried out on a $0.8 \mathrm{GHz}$ IV Pentium, and running Windows XP. For models (2.4 and 2.5 ) the capacity $W_{t}$ used by emergency surgery in each period is a stochastic parameter normally distributed.

\section{Case study}

Kashani hospital is one of the greatest Isfahan's non-profit hospitals, with 500 beds and 40 ORs. One of the greatest problems that Kashani hospital is facing is shortage in some expensive equipment, like C-ARM. This equipment is unique and most of the time scheduling of this equipment is really challenging and critical for the hospital. In this paper we attempt to schedule one of the unique equipment in operating theater, which is very useful for most of the operations. Scheduling prevents cancellations caused by overlaps in different surgeries, increases the amount of surgery operations, and omits gapes and spare times. Sometimes some operations are emergency and any kind of delay in these cases may lead to death of the patient. This aspect of the problem is more important than the financial aspect, and is the most important reason in scheduling this problem.

Different solution methods were experimented to solve this problem and make an improvement in operating theater. The numbers of periods $\mathrm{H}$ are different and are shown in Tables 1, 2 and 3. The aggregated ORs' regular capacity $T_{t}$ in a period $t$ depends on the number of available ORs (i.e. the problem size) and the regular capacity of each OR in period $t$. In this case study according to the collected data from Isfahan Kashani hospital, it is found that regular capacity of each OR in period $\mathrm{t}$ is $8 \mathrm{~h}$, and the number of available ORs is same in all periods over the planning horizon. The aggregated regular capacity is then $T_{t}=$ number of available ORs $\times 8 \mathrm{~h}$. Then, for a problem with 8 ORs, for example, the ORs' regular capacity will be $64 \mathrm{~h}$.

Durations of elective surgery are randomly and uniformly generated from the interval 0.5 to $3 \mathrm{~h}$. From collected data, it is shown that duration of each C-ARM performance for most of the operations is $20 \mathrm{~min}$, depending on the type of surgery, we may use this equipment for several times. For example for most of the spinal operations C-ARM is used for four times and each performance duration is about $10 \mathrm{~min}$, so, $q_{i}=4$ and $K=10 \mathrm{~min}$. The average daily capacity $E\left[W_{t}\right]$ needed for emergency cases depends on the number of available ORs, because $W_{t}$ is normally distributed, mean and deviation standard of normal distribution is used.

Data are collected for 125 patients from Kashani hospital in Isfahan in 30 days. $\mathrm{CE}_{i t}$ Distribution and $\mathrm{CUE}_{i t}$ Distribution are shown in "Appendix in Table 4", and $\mathrm{Co}_{t}$ has an average in these 30 days $\mathrm{Co}_{t}=500$.

It is obvious that $\mathrm{CUE}_{i t}$ is independent from $\mathrm{CE}_{i t}$ for each patient.

The number of elective cases is determined such that the workload of ORs due to elective cases is $s \%$ of the regular capacity of the entire planning horizon. In these experiments we consider problems with $s$ equal to 85 and $100 \%$. The workload of ORs due to emergency surgeries is $15 \%$ of the regular capacity of the entire planning horizon. So, when $s=85 \%$, elective cases and emergency surgeries sum up to an average of $100 \%$ regular capacity of the ORs, and when $s=100 \%$ elective and emergency surgeries sum up to $115 \%$ of ORs' regular capacity. If it is not explicitly mentioned $s$ is supposed to be equal to $85 \%$.

$\mathrm{CUE}_{i t}=\left(D_{j}+\right.$ utility $\quad$ cost + maintenance cost + Operator salary + interest rate of C-arm First cost) for patient $i$ in period $t$.

where;

$D_{j} \quad 1 / N^{(P-S)}$

$D_{j} \quad$ Depreciation expense on $j$ year

$N \quad$ Number of age

$P \quad$ First cost

$S \quad$ Salvage value

$\mathrm{CE}_{i t}=($ Drug + hospital staff (Surgeon, nurse, operators...) + clinical services (patient preparation, clinical tests....) + the tariff treatment charge) for patient $i$ ' in period $t$.

If case $i$ must be performed before period $\mathrm{Li}$, this constraint can be taken in account by choosing large costs $\mathrm{CE}_{i t}$ for $t>L i$.

To take into account cases with $B_{i}=1$ that were postponed from the previous plan, we introduced a new variable $B_{i}^{\prime}$, the effective earliest period of case $\mathrm{i}$ (or effective release period). $B^{\prime}{ }_{i}$ can take negative values. The earliest dates $B_{i}$ were generated in two steps as follows. First, we generated for each case $i$ the effective earliest date $B_{i}^{\prime}$. The $B_{i}^{\prime} s$ are integer numbers randomly selected from the set $\left\{\_2, \ldots, 5\right\}$. Then, cases with zero or negative $B_{i}^{\prime}$ will have $B_{i}$ equal to 1 , while the others will have $B_{i}$ equal to $B_{i}^{\prime}\left(B_{i}=1\right.$ if $B_{i}^{\prime}<1 ; B_{i}=B_{i}^{\prime}$ otherwise $)$. The $\mathrm{CE}_{i t}$ are assumed to be increasing in $t$ for every $i$ penalty cost: 
Table 1 Computational results of SAA and DE for elective patients
Table 2 Computational results of SAA and DE for elective and emergency patients

\begin{tabular}{lllllll}
\hline Test problem & $\begin{array}{l}\text { Traditional } \\
\text { scheduling costs }\end{array}$ & SAA & $\begin{array}{l}\text { CPU } \\
\text { (Lingo) }\end{array}$ & DE & $\begin{array}{l}\text { CPU } \\
\text { (MATLAB) }\end{array}$ & GAP \\
\hline$I=5, T=1$ & 7,124 & 6,361 & 326 & 6,361 & 4.09 & 0 \\
$I=8, T=2$ & 10,802 & $9,009.3$ & 1,030 & 9,009 & 6.19 & 0 \\
$I=15, T=4$ & 17,972 & 15,210 & 3,992 & 15,231 & 6.4 & 0.14 \\
$I=20, T=4$ & 22,509 & 19,205 & 6,826 & 19,239 & 13.79 & 0.18 \\
$I=25, T=5$ & 26,363 & - & - & 22,925 & 21.32 & NA \\
$I=30, T=6$ & 33,879 & - & - & 29,982 & 18.45 & NA \\
$I=35, T=7$ & 38,470 & - & - & 35,050 & 26.01 & NA \\
$I=40, T=8$ & 45,943 & - & - & 41,390 & 27.78 & NA \\
$I=45, T=9$ & 49,787 & - & - & 45,261 & 28.82 & NA \\
$I=50, T=10$ & 56,419 & - & - & 51,290 & 30.37 & NA \\
\hline
\end{tabular}

\begin{tabular}{lllllll}
\hline Test problem & $\begin{array}{l}\text { Traditional } \\
\text { scheduling costs }\end{array}$ & SAA & $\begin{array}{l}\text { CPU } \\
\text { (Lingo) }\end{array}$ & DE & \multicolumn{1}{l}{$\begin{array}{l}\text { CPU } \\
\text { (MATLAB) }\end{array}$} & GAP \\
\hline$I=5, T=1$ & 6,708 & 6,098 & 421 & 6,098 & 4.09 & 0 \\
$I=8, T=2$ & 9,239 & 8,399 & 1,261 & 8,399 & 6.19 & 0 \\
$I=15, T=4$ & 14,792 & 13,570 & 3,992 & 13,751 & 11.14 & 0.514 \\
$I=20, T=4$ & 20,924 & 19,041 & 7,291 & 19,197 & 15.1 & 0.818 \\
$I=25, T=5$ & 27,076 & 24,516 & 23,452 & 24,750 & 21.78 & 0.954 \\
$I=30, T=6$ & 33,133 & - & - & 30,286 & 29.63 & NA \\
$I=35, T=7$ & 38,250 & - & - & 35,060 & 34.21 & NA \\
$I=40, T=8$ & 44,633 & - & - & 40,948 & 40.2 & NA \\
$I=45, T=9$ & 49,074 & - & - & 45,147 & 48.63 & NA \\
$I=50, T=10$ & 55,462 & - & - & 51,259 & 64.05 & NA \\
\hline
\end{tabular}

\begin{tabular}{lllllll}
\hline Test problem & $\begin{array}{l}\text { Traditional } \\
\text { scheduling costs }\end{array}$ & SAA & $\begin{array}{l}\text { CPU } \\
\text { (Lingo) }\end{array}$ & DE & \multicolumn{1}{l}{$\begin{array}{l}\text { CPU } \\
\text { (MATLAB) }\end{array}$} & GAP \\
\hline$I=5, T=1$ & 6,708 & 5,034 & 394 & 5,034 & 3.95 & 0 \\
$I=8, T=2$ & 9,239 & 8,917 & 1,954 & 8,917 & 8.01 & 0 \\
$I=15, T=4$ & 14,792 & 14,582 & 4,712 & 14,616 & 7.51 & 0.231 \\
$I=20, T=4$ & 20,924 & 19,590 & 10,862 & 19,827 & 11.91 & 1.21 \\
$I=25, T=5$ & 27,076 & 24,599 & 26,452 & 24,984 & 17.21 & 1.564 \\
$I=30, T=6$ & 33,133 & - & - & 29,831 & 26.86 & NA \\
$I=35, T=7$ & 38,250 & - & - & 35,793 & 30.01 & NA \\
$I=40, T=8$ & 44,633 & - & - & 40,148 & 39.95 & NA \\
$I=45, T=9$ & 49,074 & - & - & 47,092 & 53.38 & NA \\
$I=50, T=10$ & 55,462 & - & - & 5,1528 & 69.02 & NA \\
\hline
\end{tabular}

Table 3 Computational results of SAA and DE for elective and emergency patients with considering surgeon's coefficient factor the performance of the different optimization methods, for model 2.3. and the results for models 2.4 and 2.5 are presented in Tables 2 and 3 respectively.

Results of SAA optimization method and DE are compared and traditional scheduling costs are shown in Tables 1, 2 and 3, also. It is obvious that exact solution has more precise results; but as it is shown in the Tables 1, 2 and 3, SAA is not a good choice for large size problems. As 
Fig. 2 Numerical results of elective patients

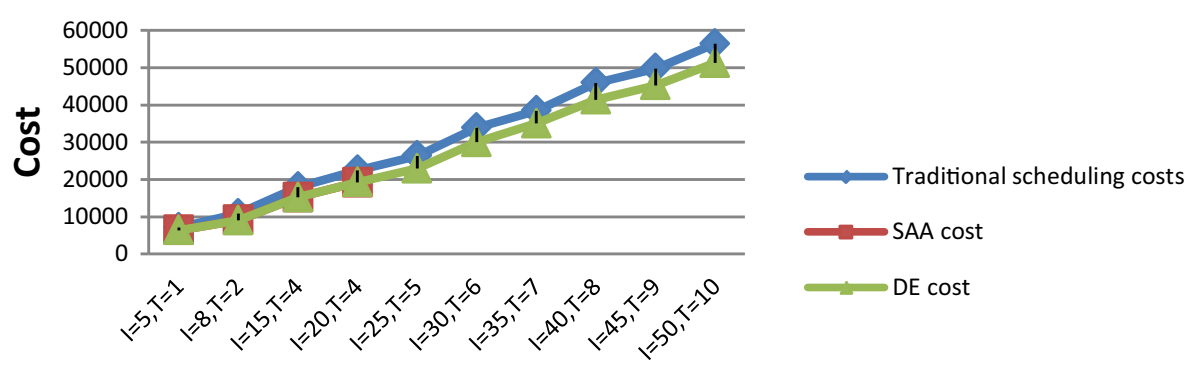

Number of patients and periods

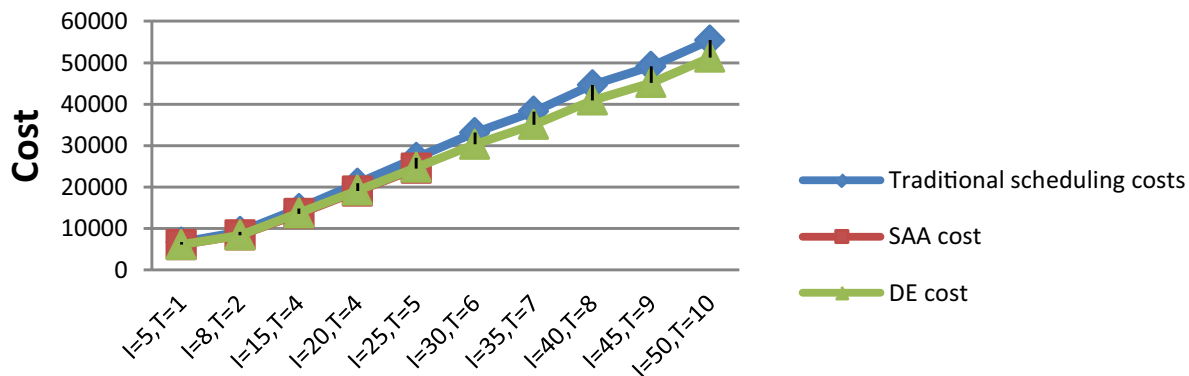

Number of patients and periods

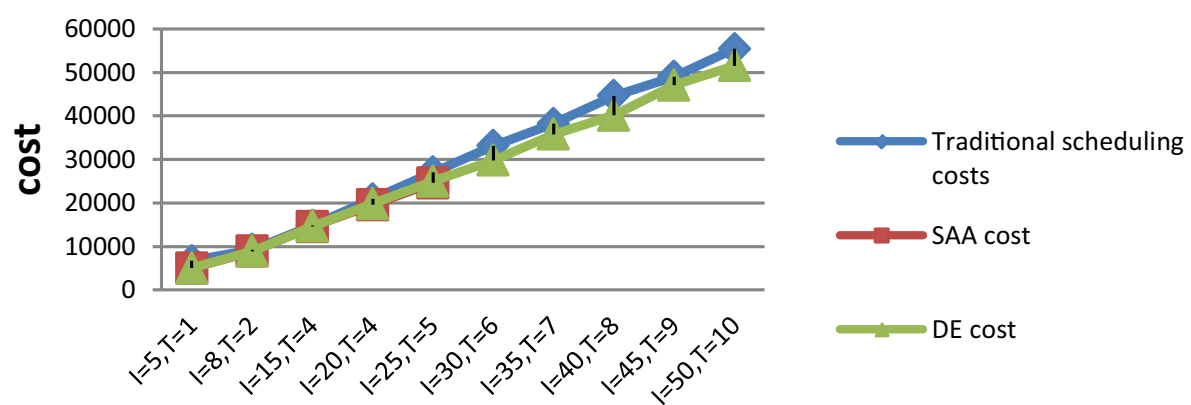

Fig. 4 Numerical Results of emergency and elective patients with considering surgeon's coefficient factor
Fig. 3 Numerical results of elective and emergency

\section{Number of patients and periods}

most of the hospital's policies are on long-term scheduling, we are forced to have long-term scheduling so we propose to implement Metaheuristic algorithm. Deviations are also shown in Tables 1, 2 and 3 . These Tables 1, 2 and 3 yield a good reduction of ORs cost. As the performances of Metaheuristic (DE) method deteriorate; deviations to the best solution increase.

Figure 2 demonstrates how the objective values of the SAA and DE change. As the number of ORs (problem size) increases, performances of Metaheuristic (DE) method deteriorate; deviations to the best solution increase and Figs. 2, 3 and 4 summarize corresponding results in detail. In Fig. 2 we have SAA cost just for 4 days, and in Figs. 3 and 4 we have SAA cost just for 5 days. Exact method (SAA) is not implementable for long periods. As hospital policies are for long periods scheduling, we are forced to have a program for long period scheduling. So we propose a Metaheuristic algorithm for long scheduling policies.

\section{Conclusions and future research}

In this paper we studied and supervised Kashani hospital Operating Theater and identified one important problem, we took it under consideration and collected data for 6 months. Then several optimization methods were proposed and compared for the elective surgery planning problem when OR capacity is shared among elective and emergency surgery and there is a unique equipment in hospital for operating theater. The planning problem has been formulated as a stochastic optimization problem. An "exact" solution method was presented and was compared with Metaheuristic method. 
Exact method is very useful for small population, but does not work properly for large size problems. As our paper is a case study paper, we proved that by implementing this stochastic model in Isfahan kashani Hospital, we will have a good reduction in overall cost. According to our experiments, if it is possible for hospitals to have short horizon planning, exact method is a very useful optimization method, and it shows that; it is a very good idea to take into account the emergency cases and also take into account surgeons coefficient factor in using this unique equipment for having better prediction in length of operations. The planning model proposed in this work is useful for hospitals using a "blocked" advance scheduling system, which reserve blocks of OR time to surgical specialties. Each specialty serving elective and emergency surgery demand can use the proposed model for the planning of electives' cases. Extension of the model to take into account various real-world constraints such as limited overtime capacity, assignment of patient to ORs, different types of ORs, random operating time is a direction of future research.

Open Access This article is distributed under the terms of the Creative Commons Attribution License which permits any use, distribution, and reproduction in any medium, provided the original author(s) and the source are credited.

\section{Appendix}

See Table 4.

Table $4 \mathrm{CE}_{i t}$ distribution and $\mathrm{CUE}_{i t}$ distribution

\begin{tabular}{|c|c|c|c|c|c|c|}
\hline \multicolumn{7}{|c|}{$\mathrm{CE}_{i t}$ distribution for 30 days } \\
\hline Day 1 & 310 & 380 & 560 & 770 & & \\
\hline Day 2 & 370 & 440 & 490 & 600 & & \\
\hline Day 3 & 480 & 550 & 660 & 740 & & \\
\hline Day 4 & 400 & 480 & 590 & & & \\
\hline Day 5 & 510 & 560 & 600 & 660 & & \\
\hline Day 6 & 570 & 630 & 650 & 710 & & \\
\hline Day 7 & 580 & 590 & 630 & 670 & 690 & 750 \\
\hline Day 8 & 480 & 510 & 550 & 670 & 720 & \\
\hline Day 9 & 500 & 530 & 570 & 610 & & \\
\hline Day 10 & 550 & 640 & 710 & & & \\
\hline Day 11 & 530 & 670 & 690 & 810 & & \\
\hline Day 12 & 600 & 630 & 680 & 780 & & \\
\hline Day 13 & 500 & 540 & 590 & 710 & & \\
\hline Day 14 & 520 & 560 & 700 & 730 & 750 & \\
\hline Day 15 & 600 & 620 & 670 & 720 & & \\
\hline Day 16 & 450 & 510 & 680 & 900 & & \\
\hline Day 17 & 500 & 650 & 730 & & & \\
\hline Day 18 & 610 & 670 & 700 & 780 & & \\
\hline Day 19 & 560 & 630 & 670 & 690 & & \\
\hline
\end{tabular}

Table 4 continued

\begin{tabular}{|c|c|c|c|c|c|c|}
\hline Day 20 & 440 & 560 & 770 & 790 & & \\
\hline Day 21 & 500 & 530 & 610 & 680 & & \\
\hline Day 22 & 530 & 570 & 640 & 720 & & \\
\hline Day 23 & 470 & 490 & 510 & 540 & 600 & 680 \\
\hline Day 24 & 430 & 540 & 590 & 650 & & \\
\hline Day 25 & 510 & 570 & 620 & 700 & & \\
\hline Day 26 & 500 & 540 & 580 & 610 & 720 & \\
\hline Day 27 & 600 & 630 & 670 & 750 & & \\
\hline Day 28 & 520 & 630 & 650 & 710 & & \\
\hline Day 29 & 630 & 700 & 810 & 900 & & \\
\hline Day 30 & 350 & 530 & 610 & 670 & 700 & \\
\hline \multicolumn{7}{|c|}{$\mathrm{CUE}_{i t}$ distribution for 30 days } \\
\hline Day 1 & 165 & 170 & 144 & 158 & & \\
\hline Day 2 & 162 & 172 & 176 & 168 & & \\
\hline Day 3 & 166 & 170 & 166 & 174 & & \\
\hline Day 4 & 140 & 132 & 159 & & & \\
\hline Day 5 & 154 & 142 & 148 & 166 & & \\
\hline Day 6 & 136 & 147 & 142 & 158 & & \\
\hline Day 7 & 139 & 156 & 171 & 134 & 157 & 160 \\
\hline Day 8 & 132 & 138 & 0 & 142 & 151 & \\
\hline Day 9 & 138 & 156 & 157 & 138 & & \\
\hline Day 10 & 160 & 148 & 162 & & & \\
\hline Day 11 & 147 & 151 & 136 & 0 & & \\
\hline Day 12 & 147 & 154 & 150 & 142 & & \\
\hline Day 13 & 154 & 145 & 142 & 138 & & \\
\hline Day 14 & 146 & 156 & 0 & 134 & 162 & \\
\hline Day 15 & 150 & 148 & 156 & 164 & & \\
\hline Day 16 & 142 & 0 & 154 & 164 & & \\
\hline Day 17 & 148 & 152 & 170 & & & \\
\hline Day 18 & 0 & 160 & 173 & 154 & & \\
\hline Day 19 & 150 & 160 & 148 & 157 & & \\
\hline Day 20 & 0 & 146 & 154 & 160 & & \\
\hline Day 21 & 150 & 143 & 148 & 154 & & \\
\hline Day 22 & 153 & 157 & 164 & 172 & & \\
\hline Day 23 & 152 & 150 & 144 & 0 & 0 & 175 \\
\hline Day 24 & 136 & 148 & 150 & 154 & & \\
\hline Day 25 & 144 & 147 & 158 & 162 & & \\
\hline Day 26 & 147 & 0 & 156 & 142 & 164 & \\
\hline Day 27 & 137 & 149 & 152 & 138 & & \\
\hline Day 28 & 146 & 154 & 146 & 148 & & \\
\hline Day 29 & 154 & 166 & 0 & 148 & & \\
\hline Day 30 & 0 & 146 & 150 & 150 & 162 & \\
\hline
\end{tabular}

\section{References}

Agnetis A, Coppi A, Corsini M, Dellino G, Meloni C, Pranzo M (2012) Long term evaluation of operating theater planning policies. Oper Res Health Care 1:95-104

Alkhabbaz A, M'Hallah R (2013) Scheduling of nurses: a case study of a Kuwaiti health care unit. Oper Res Health Care 2:1-19 
Brest J, Greiner S, Boskovic B, Memik M, Zumer V (2006) Selfadapting control parameters in differential evolution: a comparative study on numerical benchmark functions. IEEE Trans Evolut Comput 10(6):646-657

Chaabane S (2004) Gestionprédictive des blocsopératoires.Ph.D. Thesis, Institut National des Sciences Appliquées de Lyon

Civicioglu P (2011) Transforming geocentric cartesian coordinates to geodetic coordinates by using differential search algorithm. Comput Geosci 46:229-247. doi:10.1016/j.cageo.2011.12.011

Denton B, Gupta D (2003) A sequential bounding approach for optimal appointment scheduling. IIE Trans 35:1003-1016

Dexter F, Macario A, Traub RD (1999a) Which algorithm for scheduling add-on elective cases to maximizes operating room utilization? Use of bin packing algorithms and fuzzy constraints in operating room management. Anesthesiology 91:1491-1500

Dexter F, Macario A, Traub RD, Hopwood M, Lubarsky DA (1999b) An operating room scheduling strategy to maximize the use of operating room block time: computer simulation of patient scheduling and survey of patients preferences for surgical waiting time. Anesth Analg 89(1):7-20

Garey MR, Johnson MR (1979) Computers and Intractability: a guide to the theory of NP-completeness. Freeman, San Francisco

Gerchak Y, Gupta D, Henig M (1996) Reservation planning for elective surgery under uncertain demand for emergency surgery. Manage Sci 42(3):321-334

Guinet A, Chaabane S (2003) Operating theatre planning. Int J Prod Econ 85(1):69-81

Jackson RL (2002) The business of surgery: managing the OR as a profit center requires more than just IT. It requires a profitmaking mindset, too-Operating Room Info Systems, Health Management Technology

Jebali A, Hadjalouane AB, Ladet P (2005) Operating rooms scheduling. Int J Prod Econ 99:52-62

Lakshimi C, SivaKumarAppa I (2013) Application of queueing theory in health care: a literature review. Oper Res Health Care 2:25-39

Lamiri M, Xie X, Dolgui A, Grimaud F (2008) A Stochastic model for operating room planning with elective and emergency demand for surgery. Eur J Oper Res 185:1026-1037
Liu J, Lampinen J (2005) A fuzzy adaptive differential evolution algorithm”. Soft Computing 9(6) 448-462

Macario A, Vitz TS, Dunn B, McDonald T (1995) Where are the costs in peri-operative care? Analysis of hospital costs and charges for inpatient surgical care. Anesthesiology 83(6):418-433

Magerlein JM, Martin JB (1978) Surgical demand scheduling: a review. Health Serv Res 13(4):418-433

Marcon E, Kharaja S, Simmonet G (2003) The operating theatre scheduling: An approach centered on the follow-up of the risk of no realization of the planning. Int J Prod Econ 85(1):83-90

Price K, Storn RM, Lampinen JA (2005) Differential evolution: a practical approach to global optimization. Springer. ISBN:978-3540-20950-8

Qin AK, Suganthan PN (2005) Self-adaptive differential evolution algorithm for numerical optimization. In: Proceedings of theIEEE congress on evolutionary computation (CEC). pp 1785-1791

Rocca P, Oliveri G, Massa A (2011) Differential evolution as applied to electromagnetics. IEEE Antennas Propag Mag 53(1):38-49

Shapiro A, Homem-de-Mello T (2001) On the rate of convergence of optimal solutions of Monte Carlo approximations of stochastic programs. SIAM J Optim 11(1):70-86

Shapiro A, Homem de-Mello T, Kim J (2002) Conditioning of convex piecewise linear stochastic programs. Math Progr 94:1-19

Shukla RK, Ketcham JS, Ozcan YA (1990) Comparison of subjective versus data base approaches for improving efficiency ofoperating room scheduling. Health Serv Manag Res 3:74-81

Storn R (1996) On the usage of differential evolution for function optimization. In: Biennial Conference of the North American Fuzzy Information Processing Society (NAFIPS). pp 519-523

Tao G, Zhao K, Gift T, Qiu F, Chen G (2012) Using a resource allocation model to guide better local sexually transmitted disease control and prevention programs. Oper Res Health Care $1: 23-29$

Weiss EN (1990) Models for determining estimated start times and case orderings in hospital operating rooms. IIE Trans 22(2):143-150

Zhou J, Dexter F (1998) Method to assist in the scheduling of add-on surgical cases, upper prediction bounds for surgical case durations based on the log-normal distribution. Anesthesiology 89(5):1228-1232 